\title{
Laser Triangulation System for the Measurement of Volume and Color of Wounds
}

\author{
Urban PAVLOVČIČ, Matija JEZERŠEK, Janez MOŽINA \\ Faculty of Mechanical Engineering, University of Ljubljana, Ljubljana, Slovenia
}

\begin{abstract}
A compact and portable rotational laser profilometer was developed for accurate measurement of the volume and color of wounds. The measuring system is based on the principle of laser-line triangulation and rotary motion along the measuring surface. The result is a 3D shape of an object, in color. The system is calibrated by measuring the surface of a reference object. The standard deviation of differences between the reference surface and the measurement was $0.4 \mathrm{~mm}$ after calculation of transformation parameters. The measuring range is $150 \times 150 \times 200 \mathrm{~mm}$. The developed software is used for data acquisition and analysis. It is a tool for semi-automatic wound edge determination based on color variations using NURBS surface for the virtual healthy skin approximation. Once the wound edge is determined based on the color information, only the partial volumes within the wound boundaries are considered. In this way accuracy is significantly improved. The algorithm for automatic determination of the area of virtual healthy skin is based on the previously found wound edge. The results on the measurements of in-vivo wounds indicate that the repeatability is mostly dependent on the determination of the virtual healthy skin. By using this novel approach the relative error of the calculated volume was reduced under $10 \%$.
\end{abstract}

Keywords: 3D measurement, surface digitalization, profilometry, laser triangulation, image processing, wound shape.

\section{Introduction}

In medicine, there is a need to measure wound dimensions as predictive factors of venous leg ulcer, pressure sores or wounds healing [1-3]. Venous leg ulcers usually occur on the lower leg area of elderly people, who have problems with the chronic venous insufficiency. With a view to objectively monitor the treatment progress and to adapt it consequently if needed, there is a necessity for measuring methods to measure dimensions and picture the bottom color of the venous leg ulcer. Conventional methods and equipment are used such as measuring tapes, calipers, plastic foils, on which wound shape can be copied, etc...[3, 4].Recently, optical three-dimensional measuring systems are used for non-contact measurement of all wound-shape characteristics (circumference, area and volume). By using the existing, although inaccurate methods, the length, width and depth can be measured [4-6].

There are many different approaches to the non-contact measurement. Structured light triangulation measuring systems are presented in [6-9]. Hand-held laser-based 3D measuring system is presented in [6], consisting of a laser projector and a commercial digital camera. Light pattern of 33 laser lines are projected onto the measured surface. Measuring range of the system is $100 \times 100 \times 100 \mathrm{~mm}$, acquisition time $5 \mathrm{~ms}$ and accuracy is $0.07 \mathrm{~mm}$. Thermal imaging device was added to the measuring system in [7], which is able to acquire geometry, texture and temperature data of the surface. Wound edge is determined on image, combined with visible and thermal image. The system in [8] captures two images; first, when surface is illuminated with homogenous, bright light and the second, when color coded parallel stripes are projected to the surface. The data acquisition procedure is $0.5 \mathrm{~s}$ long. The wound edge is determined on the basis of red-green ratio difference between the wound and the healthy skin. The accuracy of the system is $0.4 \mathrm{~mm}$ when human skin is measured. Virtual healthy skin $(\mathrm{ViHS})$ is approximated by using third order cubic splines interpolated over the ulcer. Stereophotogrammetric principle of the wound geometry measurement is used in [10], where three cameras are placed in a triangle at a distance of $15 \mathrm{~cm}$ between each other.

The presented system is also based on structured light triangulation, using only one laser stripe. On the basis of past experiences [6] the significance of wound color was noted. The surface color measurement was added for that reason. The software for semi automatic wound edge and ViHS determination was developed to improve the repeatability of wound analysis. 


\section{Measuring system}

The measuring system is based on the principle of laser line triangulation and rotary motion along the measuring surface. It has a measuring range of 150x150x200 mm. Color camera (PointGrey, FireFly MV, resolution 640x480 pix, sensor size 1/3"), laser-line projector (World Star Tech, power 3,5mW, wavelength $635 \mathrm{~nm}$, spread angle $15^{\circ}$ and line width $<1 \mathrm{~mm}$ ) and rotary mechanism consisting of housing, swingarm and linear stepper motor are the main components of the measuring system (see Figure 1). The acquisition process has two consecutive phases; geometry measurement and color measurement. The laser light plane is scanned towards the measured surface during the first phase. Intersecting curve is formed on the interception of the laser plane and the surface. It is observed with camera placed on the other side of the swingarm (see Figure 2). Acquired images are processed by the sub-pixel line detection algorithm [16]. Three-dimensional coordinates of the points of the interception curve are then calculated according to known image coordinates, swingarm angle, triangulation angle, projector-to-sensor distance and camera intrinsic parameters (lens focal length, pixel dimensions and distortion) [17].

The surface color is measured during the second phase - in return stroke. The laser projector is turned off and the camera is adjusted to less intense light conditions. The red, green and blue values of previously detected pixels are stored for each interception curve.

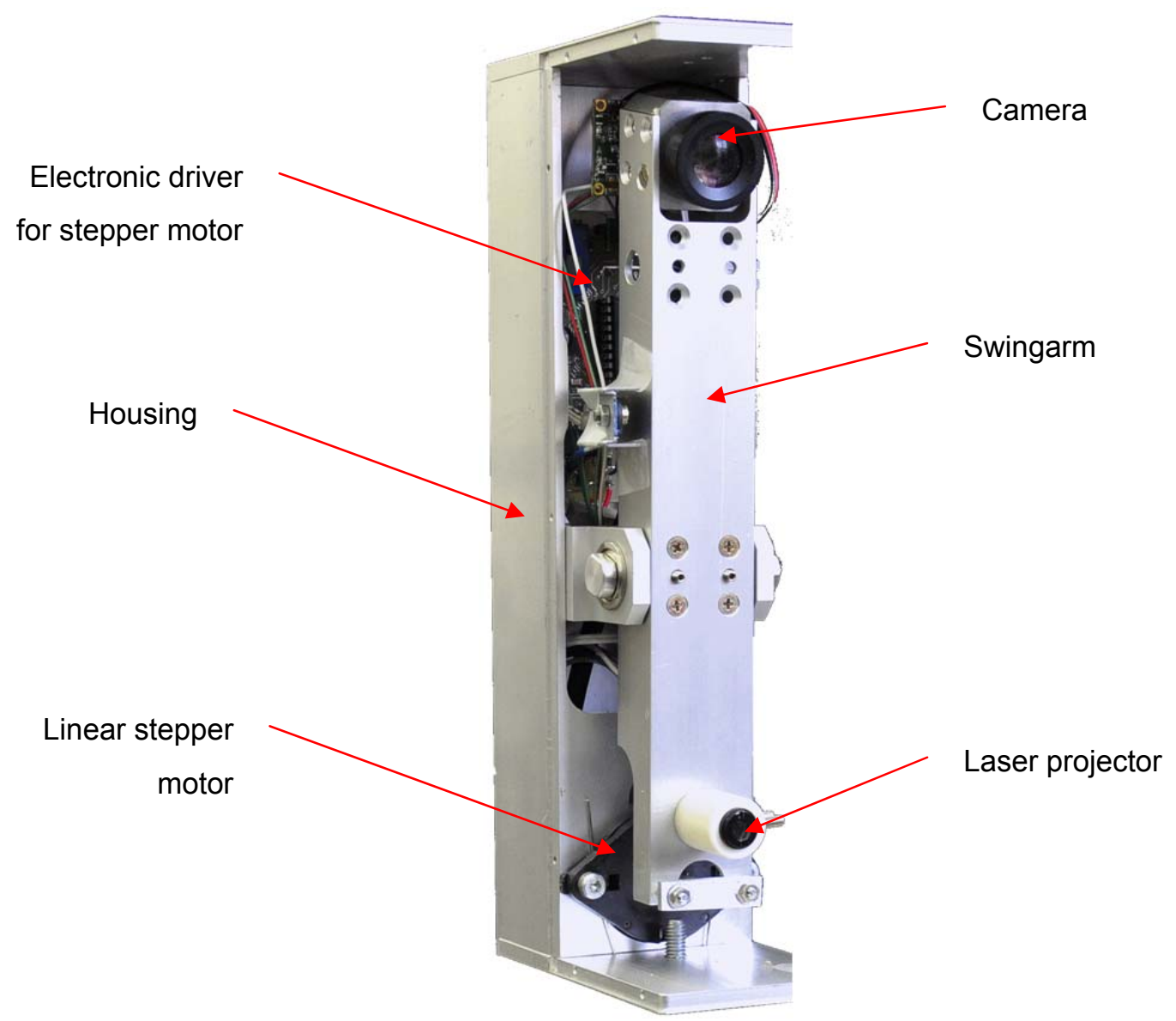

Fig. 1. Measuring system (with removed cover). 


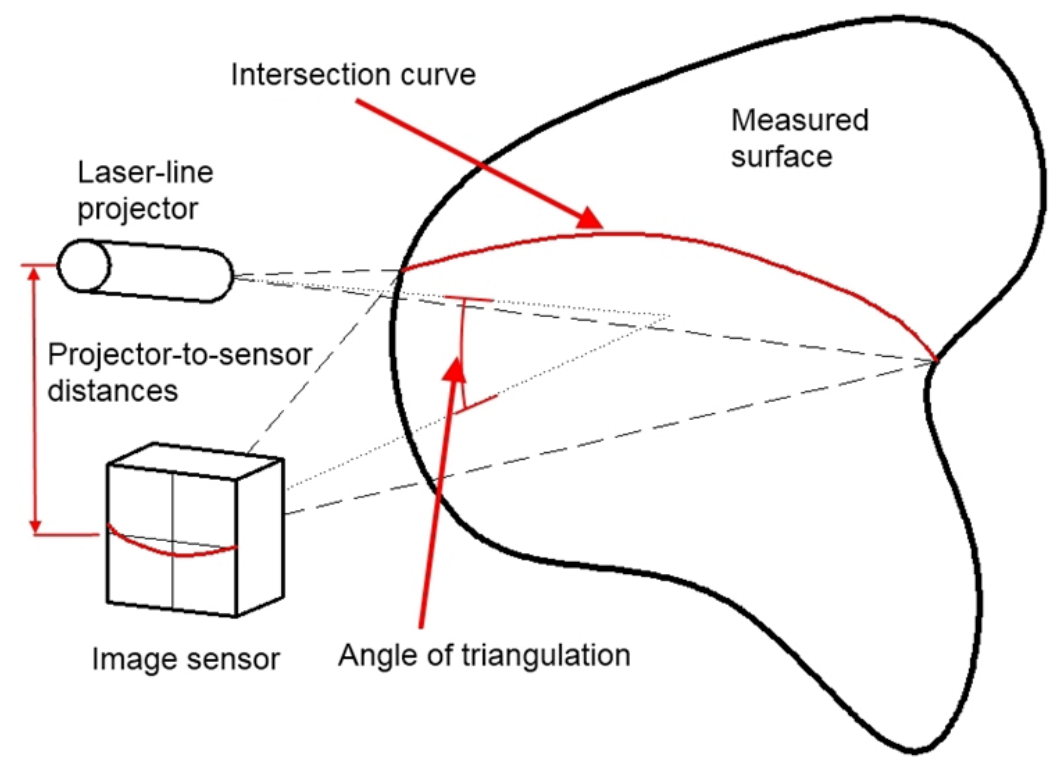

Fig 2. Schematic of laser line triangulation principle.

\section{Calibration}

The calibration procedure consists of measuring the reference geometry and calculation of the system transformation parameters such as camera position and orientation, its focal length, pixel size and distortion, etc.

The calibration set-up is seen on the left part of figure 3 . The reference geometry plate is fixed on a translation table. The plate is oriented perpendicularly to the table moving direction. Eleven measurements were made across the measuring range (see Figure 3-center). The distance between the measuring system and the reference plate was between $800 \mathrm{~mm}$ and $1160 \mathrm{~mm}$, with a $36 \mathrm{~mm}$ step. The measurements were used for calculation of the above mentioned parameters, using the method with minimal displacement search between measured and reference plane [17]. The criterion for parameter evaluation is standard deviation of difference between the reference and the measured surface. The achieved accuracy of the measuring system was $0.4 \mathrm{~mm}$.

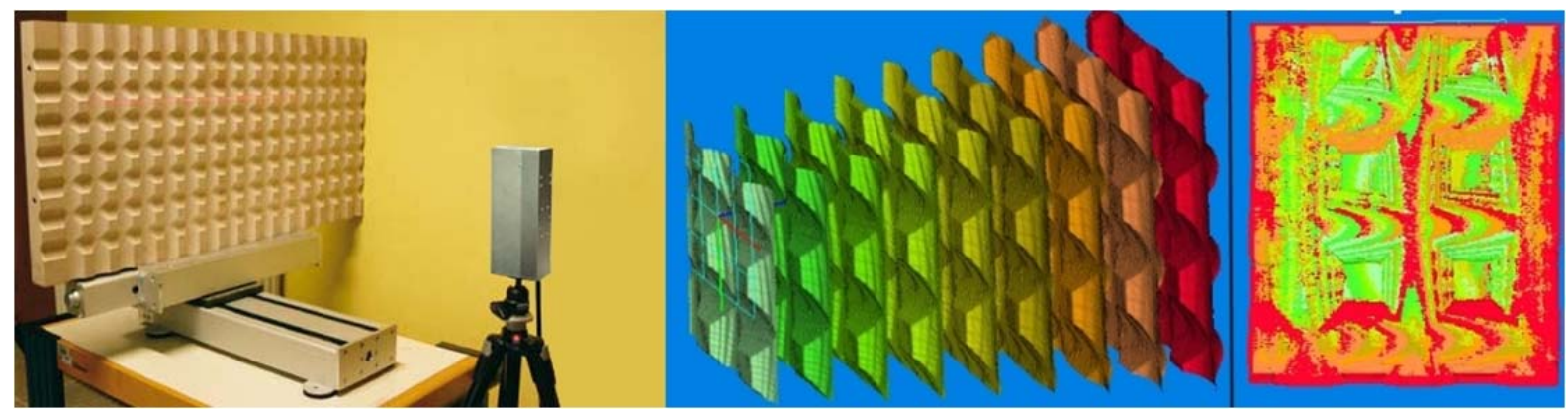

Fig. 3. Measuring reference object (left), measurements of reference object at different distances (center) and front view of displacements after the calibration

\section{Measurement analysis}

For monitoring the healing process of the venous leg ulcer it is important to evaluate its characteristics; circumference, area and volume. For this purpose software was developed, using Visual $\mathrm{C}++$ and Intel IPP, Open CV and nurbs++ libraries [14, 15].

The first step is wound edge determination. The process is divided into several steps; interim results can be seen in Figure 4. First, the most appropriate color channel (red, green or blue) is selected, where the contrast between the wound and the surrounding healthy skin is the highest. In most cases, that is the blue or the green channel. Both, the color of the human skin as well as the color of wound usually contain a lot of red component, Secondly, the wound edge candidate points are determined 
using the Canny edge detector algorithm [13]. The threshold is slightly lower than the one recommended in [13] because it was found to be much more convenient to erase redundant points than the opposite. In the next step smaller gaps in the curve are closed using functions erode and dilate consecutively. The whole area of the wound is flooded using flood-fill function. In the final phase, the edge points of flooded area are connected into a 3D polygon, which can be used in algorithms for wound circumference, area and volume calculations.

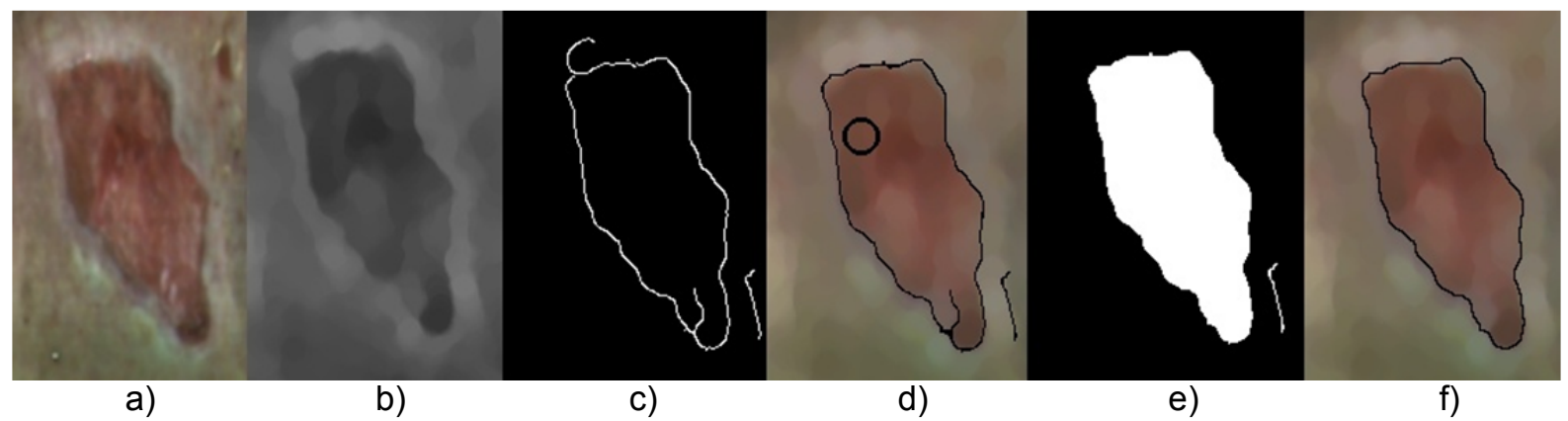

Fig. 4 The interim results of wound-edge determination. a) Source image. b) Filtered blue channel. c) Detected edge. d) Manual edge drawing and deletion. e) Filled wound-edge area. f) Final result

Wound volume is determined as a volume between the measurement and the virtual healthy skin (ViHS). ViHS is approximated by NURBS surface (see Figure 5), which is constructed as a blend between four boundary curves [12]. These curves are splines, approximated by points of the measured surface between neighboring vertex points. The difference between the measured surface and the $\mathrm{ViHS}$ is then calculated and wound volume is determined by numerical integration over the entire wound area.

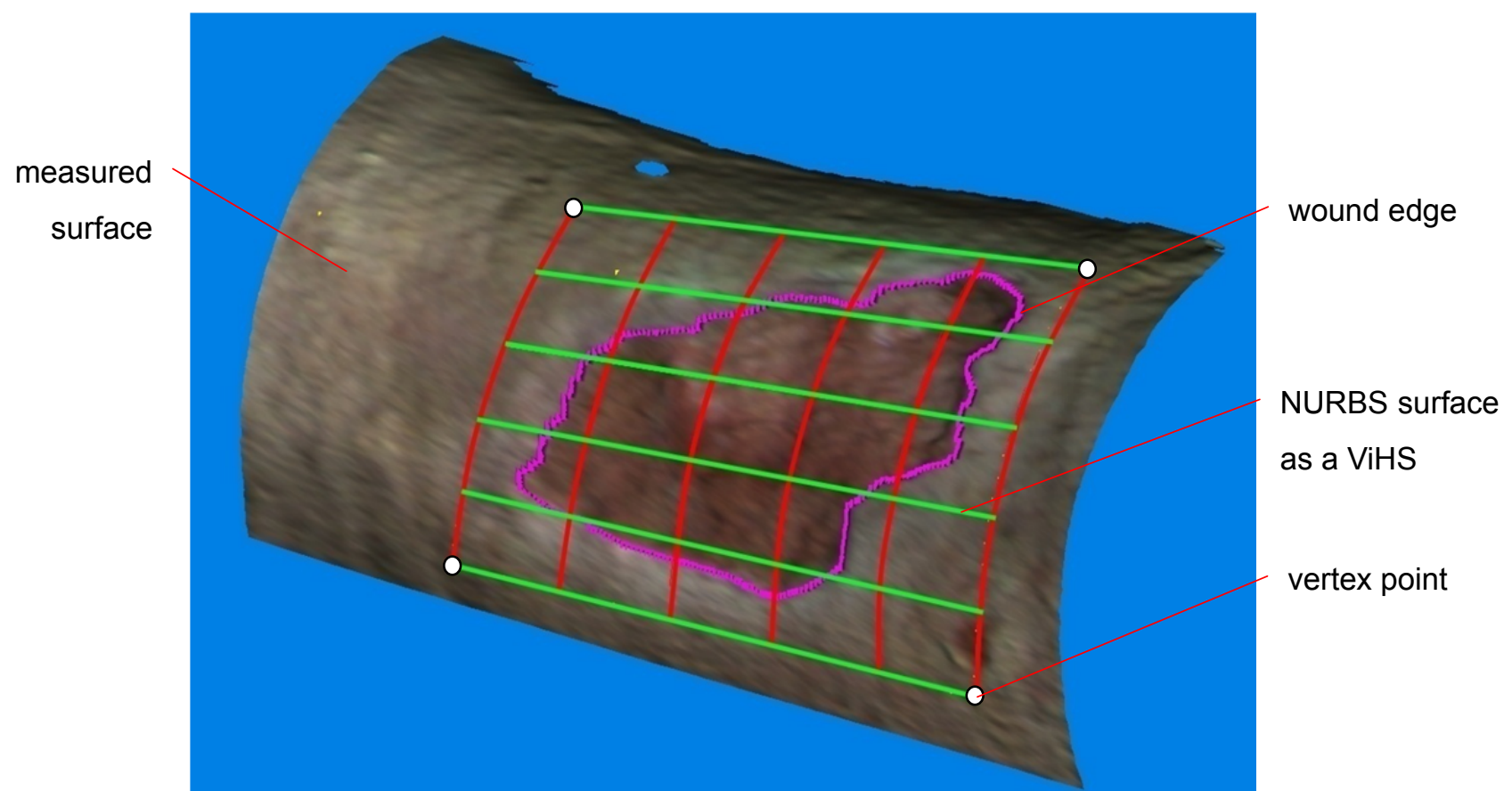

Fig. 5.Measured surface with determined wound-edge (pink dots) and approximated virtual healthy skin (green and red lines)

Due to great influence of the approximated ViHS on the final results, it is important to make the approximation process as repeatable as possible. The algorithm for automatic vertex points determination was introduced for that reason. It is based on the principle of outlining the wound edge with a rectangle having minimal area. The rectangle is enlarged for $20 \%$ and it's vertex points are used for ViHS construction. 


\section{Measurements}

The in-vivo measurements of the venous leg ulcers were conducted in four-week intervals in cooperation with Department of Dermatovenereology, University Medical Centre Ljubljana. Some of the measured wounds are shown in figure 6 . Wound edge, determined using the described procedure, is also shown. It can be observed that the measurements are quite detailed. During the analysis of the geometric characteristics of the wound we were trying to determine the various causes of error, when the analysis is performed multiple times on the same data. It turned out that the wound edge determination is very repeatable and it makes a small contribution to the total error. At constant approximated ViHS surface and different wound edge calculations the volume varied by $1 \%$, the area by $2 \%$ and the circumference by $0,5 \%$. Contrary to that, when the wound edge is held constant and the approximated $\mathrm{ViHS}$ is varied, the results may vary by as much as $150 \%$. The cause of such large deviations is the shape of the surrounding surface. A minimal change in the position of vertex points can significantly change the boundary conditions of the surface approximation and consequently the shape of the ViHS. For that reason the algorithm for automatic vertex determination was developed. The results indicate decrease of error, the value depending on the location of the wound.

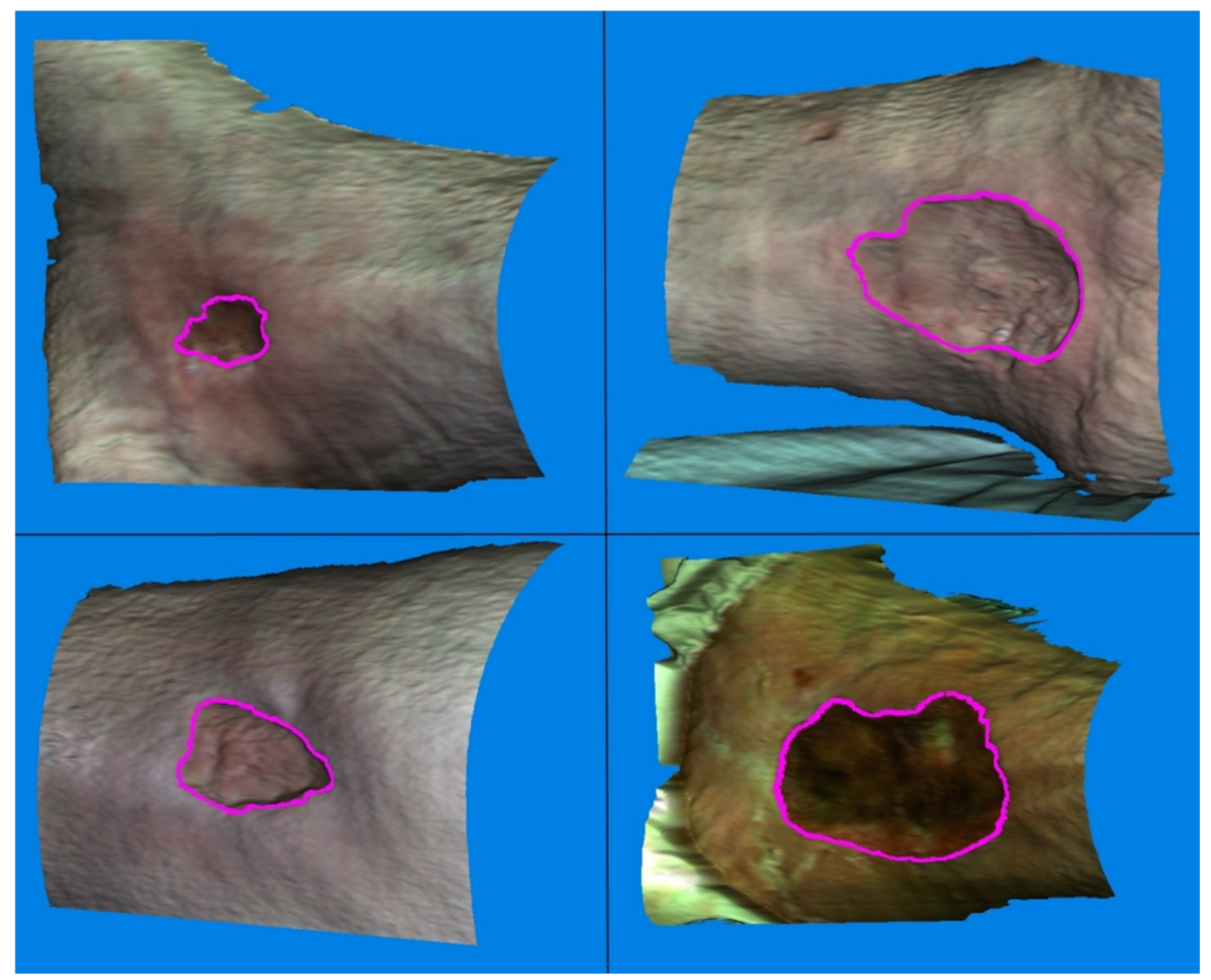

Fig. 6. Test measurements with determined edge (pink dots).

\section{Conclusion}

A laser rotary profilometer and software for the measurement analysis was developed. After calibration, the standard deviation of differences between the reference model and the measurement was $0.4 \mathrm{~mm}$. The measuring system enables measuring 3D surfaces in color a basis for wound edge semi-automatic determination. Virtual healthy skin (ViHS) is approximated by NURBS surface. The results of the analysis performed on the measurements of in-vivo wounds indicate that the repeatability is mostly dependent on the determination of the virtual healthy skin. The influence of the surrounding skin shape is substantial: if the surrounding skin is undulating a minimal change in the vertex point determination can lead to a significant change of boundary conditions for ViHS approximation. The relative error of the calculated volume was reduced under $10 \%$ by using the procedure for automatic vertex determination. 
The analysis also showed that the use of NURBS curves for ViHS approximation in not appropriate for all parts of the leg; it is particularly problematic on the area around the ankle bone and toe mounds. Further development will focus on optimizing approximation algorithms.

\section{References}

1. Moore K, McCallion R, Searle RJ, Stacey MC,Harding KG., (2006): Prediction and monitoring the therapeutic response of chronic dermal wounds. Int Wound J, Vol. 3, pp. 89-96.

2. Plassman P., Melhuish J., Harding K., (1995): Problems of assessing wound size. Wound Healing Research Unit, University of Wales College of Medicine, Cardiff CF4 4XN, Wales, United Kingdom.

3. Kecelj Leskovec N., Pohar Perme M., Jezeršek M., Možina J., Pavlović M. D., Lunder T., (2008): Initial healing rates as predictive factors of venous leg ulcer healing: the use of a laser-based 3-D ulcer measurement, Wound Repair Regen, Vol. 18, pp. 507-512.

4. Flanagan M., (2003): Wound measurement: can it help us to monitor progression to healing?, J Wound Care, Vol. 12, No. 5, pp. 189-194.

5. Majeske C., (1992): Reliability of wound surface area measurements, Phys Ther, Vol. 72, pp. 138-141.

6. Kecelj - Leskovec N., Jezeršek M., Možina J., Pavlović M. D., Lunder T., (2007): Measurements of venous leg ulcers with a laser-based three-dimensional method compared to computer planimery with photography, Wound Rapair And Regeneration, Vol. 15, num. 5, pp. 767-771.

7. Barone S., Paoli A., Razionale A. V., (2011): Assessment of chronic wounds by three-dimensional optical imaging based on integrating geometrical, chromatic, and thermal data, Proceedings of the Institution of Mechanical Engineers, Part H: Journal of Engineering in Medicine, Vol. 225: pp. 181. (53)

8. Plassmann P.,Jones B. F. (1992): Measuring area and volume of human leg ulcers by colour coded structured light, Wound Care, Vol. 1, pp. 35-38. (55)

9. Korber A, Rietkotter J, Grabbe S, Dissemond J. (2006): Three-dimensional documentation of wound healing: first results of a new objective method for measurement. J Dtsch Dermatol Ges, Vol. 4, pp.848-854. (58)

10. Boersma S., den Heuvel F. V., Cohen A., Scholtens R., (2000): Photogrammetric wound measurement with a three-camera vision system. Int. Arch. Photogrammetry Remote Sens., Amsterdam, The Netherlands, 2000, Vol. 33, pp. 84-91. (57)

11. Albouy B., Treuillet S., Lucas Y., Pichaud J. C., (2005): Volume Estimation from Uncalibrated Views Applied to Wound Measurement," Int. Conference on Image Analysis and Processing, pp. 945-952.

12. L. Piegl (1997), The NURBS book, 2nd edition, Berlin, Springer.

13. J. Canny (1986), A Computational Approach To Edge Detection, IEEE Trans. Pattern Analysis and Machine Intelligence, Vol. 8, num. 6, pp. 679-698 .

14. OpenCV, http://opencv.willowgarage.com/wiki/, (25.1.2011).

15. Intel (2005), Integrated Performance Primitives for Intel Architecture, Reference Manual, Volume 2: Image and Video Processing, Document Number: A70805-016, http://developer.intel.com.

16. Jezeršek M, Možina J, (2003): A Laser Anamorph Profilometer, Journal of Mechanical Engineering, 49(2), 76-89.

17. Jezeršek, M., and Možina, J., (2009): "High-speed measurement of foot shape based on multiple-laser-plane triangulation", Opt. eng., Vol.48, No.11, pp.113604-1-113604-8. 